\title{
Proyecto integrador grupal como herramienta de docencia y aprendizaje en Mecatrónica
}

\author{
Boronat-Moll, Carles ${ }^{\text {a }}$ Dahoui Obon, Jose Mariano ${ }^{b}$ \\ acarbomol@,upvnet.upv.es Departamento de organización de empresas, Universitat Politècnica de València \\ b jodaob@itaca.upv.es Instituto Itaca, Universitat Politècnica de València
}

\begin{abstract}
Carrying out a group project in the second course of the higher level training cycle in Industrial Mechatronics, adapting one of the subjects (Mechatronics Systems Configuration) as well as others of the same course, results in the making of an automated prototype.

The achievement of this challenge based on project-based learning provides students with a high motivating reward for future challenges. In addition, being a group work based on learning by doing, it also develops teamwork skills and integrates most of the professional skillsof the training cycle.
\end{abstract}

Keywords: learning by doing, competences, project-based learning.

\section{Resumen}

La realización de un proyecto grupal en el segundo curso del ciclo formativo de grado superior en Mecatrónica Industrial, adaptando una de las asignaturas (Configuración de sistemas mecatrónicos) así como otras del mismo curso, da lugar a la realización de una maqueta automatizada.

La consecución de este reto basado en el aprendizaje por proyectos aporta a los alumnos una alta recompensa motivadora para futuros retos. Además, al ser un trabajo grupal basado en aprender haciendo (learning by-doing), desarrolla también competencias del trabajo en equipo e integra la mayoría de las competencias profesionales del módulo.

Palabras clave: aprender haciendo, competencias, aprendizaje basado en proyectos.

\section{Introducción}

Los estudiantes del s. XXI necesitan poder conectar los conocimientos y habilidades aprendidos académicamente y ser capaces de aplicarlos a nuevos escenarios desarrollando conocimiento tácito (Carneiro, 2007). Así son las diversas competencias necesarias para los estudiantes recogidas por Luna Scott (Scott, 2015) en su trabajo para la Unesco.

Para un aprendizaje activo y basado en aprender haciendo (learning by doing), el aprendizaje basado en proyectos se presenta como una herramienta útil. Así, según Bell (Bell, 2010), el aprendizaje por proyectos es "An innovative approach to learning that teaches a multitude of strategies critical for success in the twenty-first century. Students drive their own learning through inquiry, as well as work collaboratively to research and create projects that reflect their knowledge". Los alumnos, partiendo de sus conocimientos previos en neumática, mecánica, ajuste, dibujo, montaje, etc., adquiridos en el primer curso de su ciclo formativo, tienen que realizar una maqueta mecatrónica que funcione y desempeñe un proceso durante el 
curso lectivo y antes de la realización de la Formación en Centros de Trabajo (FCT). Además de los conocimientos previos, se añaden los conocimientos adquiridos en paralelo a la realización del proyecto y explicados en otras asignaturas, en cuento a automatización, dibujo en 3D, gestión de la calidad y mantenimiento. El profesor adquiere por tanto un papel de profesor-guía, que proporciona realimentación según la evolución del proyecto, facilita soluciones, asesora e interviene en problemas dentro del grupo cuando éste no es capaz de solucionarlos, tanto a nivel técnico como de organización y funcionamiento, pero dejando la iniciativa a los alumnos (reelaborado a partir de Blumenfeld (1991)).

\section{Objetivo}

La asignatura de Configuración de Sistemas Mecatrónicos se imparte en el segundo curso del Ciclo Formativo de Grado Superior de Mecatrónica Industrial (RD 1576/2011 y Orden 31/2015), con una duración por curso de 160 horas y 7 horas semanales.

El objetivo general de este proyecto para los alumnos es realizar una maqueta mecatrónica o prototipo electromecánico funcional antes de la terminación del curso, que en este caso dura dos trimestres, ya que en el último trimestre se realizan las FCT.

Podemos subdividir este objetivo general en otros objetivos específicos más concretos, directamente relacionados con las diferentes competencias que se van a desarrollar:

- Resolución de problemas, elección de una solución factible dado los materiales disponibles.

- Consulta de soluciones en catálogos comerciales

- Realización de bocetos, planos en 2D y en 3D, acotación de los mismos.

- Coordinación y trabajo en equipo; división de roles. Asunción de responsabilidades.

- Cableado eléctrico y de un cuadro eléctrico, planos eléctricos.

- Cableado y planos neumáticos.

- Realización de presupuestos

- Realización de una secuencia automática tipo GRAFCET, pero que funcione en la medida de lo posible de forma combinacional. Programación de la secuencia automática. Diseño e implementación de herramientas tipo Human Interface Machine para la interacción entre el operario y la máquina.

- Creatividad e innovación.

- Cumplimiento de plazos. Programación de tareas y seguimiento con las herramientas PERT y GANT.

- Elaboración de un plan de mantenimiento.

- Torneado, soldado, montaje, ajuste mecánico.

\section{Desarrollo de la innovación}

La asignatura elegida para la aplicación de este aprendizaje basado en proyectos es la de "Configuración de Sistemas Mecatrónicos" que se imparte en el segundo curso del Ciclo Formativo de Grado Superior de Mecatrónica Industrial (RD 1576/2011 y Orden 31/2015), con una duración por curso de 160 horas y 7 horas semanales.

Se ha elegido esta asignatura porque es integradora contenidos impartidos en otras asignatura y que por tanto son aplicables a un proyecto integrador como el planteado, a destacar (Orden 31/2015):

- Determinación de las características de sistemas mecatrónicos. 
- Cuadros, instalaciones mecánicas, eléctricas, neumáticas e hidráulicas.

- Condiciones de la puesta en marcha de sistemas mecatrónicos.

- $\quad$ Cadenas cinemáticas.

- Procedimientos de puesta en marcha.

- Desarrollo de soluciones constructivas de productos mecatrónicos.

- Dimensionado y selección de elementos.

- Elaboración de presupuestos

- Elaboración de documentación técnica

Mientras que las competencias profesionales y sociales del ciclo formativo a las que pretende contribuir de una mayor manera son (RD 1576/2011):

a) Obtener los datos necesarios para programar el montaje y el mantenimiento de los sistemas mecatrónicos.

b) Configurar sistemas mecatrónicos industriales, seleccionando los equipos y elementos que las componen.

c) Planificar el montaje y mantenimiento de sistemas mecatrónicos industriales: maquinaria, equipo industrial y lineas automatizadas de producción, entre otros, definiendo los recursos, los tiempos necesarios y los sistemas de control.

d) Supervisar y/o ejecutar los procesos de montaje y mantenimiento de sistemas mecatrónicos industriales, controlando los tiempos y la calidad de los resultados.

e) Supervisar los parámetros de funcionamiento de sistemas mecatrónicos industriales, utilizando instrumentos de medida y control y aplicaciones informáticas de propósito específico.

f) Diagnosticar y localizar averias y disfunciones que se produzcan en sistemas mecatrónicos industriales, aplicando técnicas operativas y procedimientos específicos, para organizar su reparación.

i) Poner a punto los equipos, después de la reparación o montaje de la instalación, efectuando las pruebas de seguridad y funcionamiento, las modificaciones y ajustes necesarios, a partir de la documentación técnica, asegurando la fiabilidad y la eficiencia energética del sistema.

k) Supervisar o ejecutar la puesta en marcha de las instalaciones, ajustando los parámetros y realizando las pruebas y verificaciones necesarias, tanto funcionales como reglamentarias.

l) Elaborar la documentación técnica y administrativa para cumplir con la reglamentación vigente, con los procesos de montaje y con el plan de mantenimiento de las instalaciones.

m) Elaborar planos y esquemas con las herramientas informáticas de diseño, para actualizar la documentación y reflejar las modificaciones realizadas.

n) Organizar, supervisar y aplicar los protocolos de seguridad y de calidad en las intervenciones que se realizan en los procesos de montaje y mantenimiento de las instalaciones.

n) Adaptarse a las nuevas situaciones laborales, manteniendo actualizados los conocimientos científicos, técnicos y tecnológicos relativos a su entorno profesional, gestionando su formación y los recursos existentes en el aprendizaje a lo largo de la vida y utilizando las tecnologías de la información y la comunicación.

o) Resolver situaciones, problemas o contingencias con iniciativa y autonomía en el ámbito de su competencia, con creatividad, innovación y espíritu de mejora en el trabajo personal y en el de los miembros del equipo. 
p) Organizar y coordinar equipos de trabajo con responsabilidad, supervisando el desarrollo del mismo, manteniendo relaciones fluidas y asumiendo el liderazgo, así como aportando soluciones a los conflictos grupales que se presenten.

q) Comunicarse con sus iguales, superiores, clientes y personas bajo su responsabilidad, utilizando vías eficaces de comunicación, transmitiendo la información o conocimientos adecuados y respetando la autonomía y competencia de las personas que intervienen en el ámbito de su trabajo.

r) Generar entornos seguros en el desarrollo de su trabajo y el de su equipo, supervisando y aplicando los procedimientos de prevención de riesgos laborales y ambientales, de acuerdo con lo establecido por la normativa y los objetivos de la empresa.

La asignatura por tanto es capaz de integrar y contribuir de una forma significativa a muchas de las competencias del ciclo formativo.

También contribuiría a las cinco unidades de competencia incluidas en la relación de cualificaciones y unidades de competencia del Catálogo Nacional que constituyen la cualificación profesional completa de Planificación, gestión y realización del mantenimiento y supervisión del montaje de maquinaria, equipo industrial y líneas automatizadas de producción (Real Decreto 182/2008).

En los siguientes puntos pasamos a describir cada una de las grandes partes en que se divide el proyecto como un ejemplo de la innovación realizada.

\subsection{Planificación}

El planteamiento inicial es dar a los alumnos un tablero liso, sin nada encima. Ellos deben empezar a plantear sus ideas al profesor, que en función de la experiencia, del material disponible y de la dificultad del proyecto dará su aprobación. Posteriormente deben pasar a la fase de boceto, realizando el acopio de materiales (y pidiendo compra del que no esté disponible y que en función del presupuesto del departamento sea posible comprar) y empezando con el montaje que debería de ir en paralelo al diseño. Más o menos un poco antes o después de navidades deberían estar realizando el cuadro eléctrico. Previamente, se habrá tomado la decisión sobre la secuencia aproximada, número y tipo aproximado de sensores. En paralelo se debería ir trabajando en la parte mecánica que debería estar terminada en un $80-90 \%$ en ese momento. A mediados de enero debería estar realizado el cuadro eléctrico y se deberían cablear los sensores y accionadores eléctricos. En paralelo, también se realiza la instalación neumática y se continúa terminando mecánicamente el proyecto y se empiezan a realizar pruebas. También por esta fecha se empieza a realizar la secuencia en forma de GRAFCET del proyecto para terminar de programar a principios de febrero, realizando un volcado en el autómata y las primeras pruebas. Durante todo febrero se deberían realizar la puesta en marcha, resolución de averías y acabado mecánico.

En paralelo, durante todo el proceso se ha documentado el diseño, planos mecánicos, neumáticos y eléctricos. Esta documentación se integra para configurar la versión en papel del proyecto grupal que acompañará al proyecto físico. La fecha final de entrega es a principios de marzo. Posteriormente se realiza una jornada de puertas abiertas en el instituto, dirigida a los alumnos del instituto, las familias de los alumnos, exalumnos, autoridades y cualquier persona interesada que pueda visitar el lugar donde se exponen los proyectos en funcionamiento. Durante la misma, los alumnos por turnos se encargan de realizar la operación de las maquetas o prototipos mecatrónicos. 


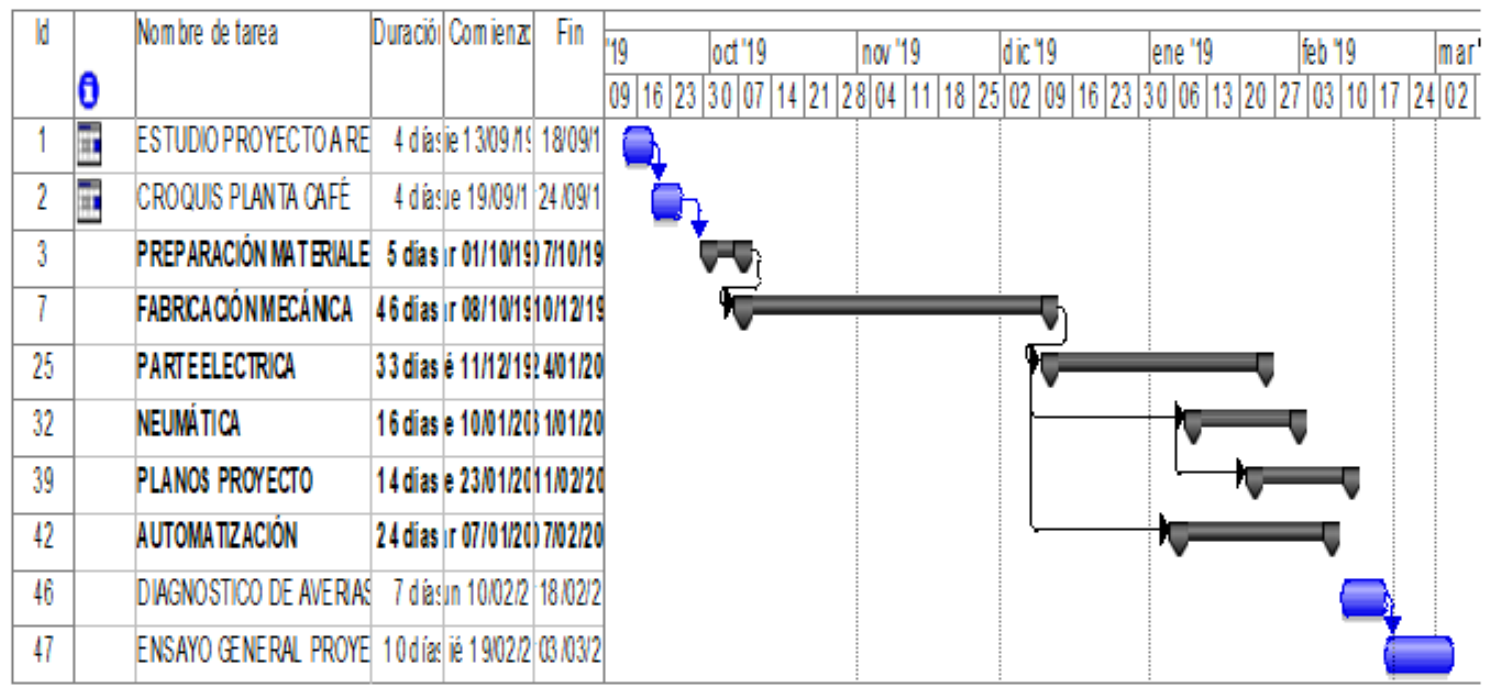

Fig. 1 Ejemplo de Gantt realizado por los alumnos

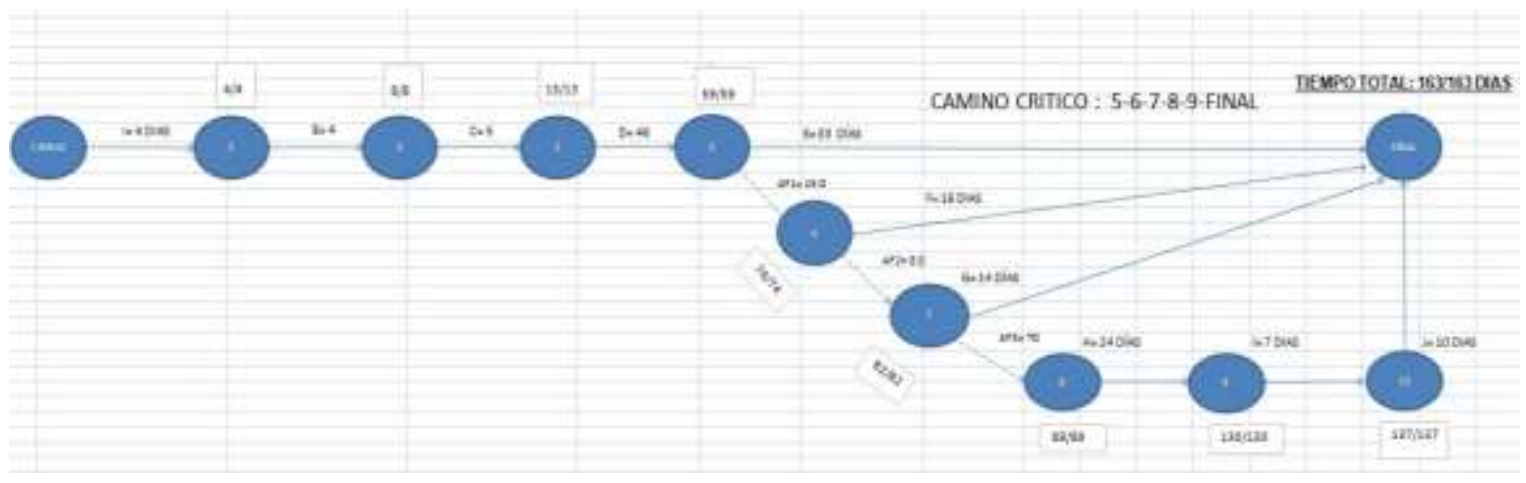

Fig. 2 Ejemplo de PERT realizado por los alumnos

\subsection{Boceto y planos mecánicos finales}

Se parte de una idea de los alumnos que debe ser aprobada por el profesor en función de su adecuación a las enseñanzas del ciclo y a la disponibilidad. Es entonces cuando se debe realizar un boceto que todavía puede sufrir modificaciones. Se trabaja siempre con el ciclo PDCA (Plan-Do-Check-Act), teniendo una elevada tolerancia al fallo dado que estamos en una fase de diseño y que los alumnos es la primera vez que realizan un proyecto similar.

Por ejemplo, no se aceptó la idea de un generador eólico tipo molino, pero si se aceptaron, un parking automatizado o una embotelladora. En general, se valora que haya una manipulación de material o piezas y que el boceto sea similar al de una línea automatizada como la que se podría encontrar en el mundo real. 


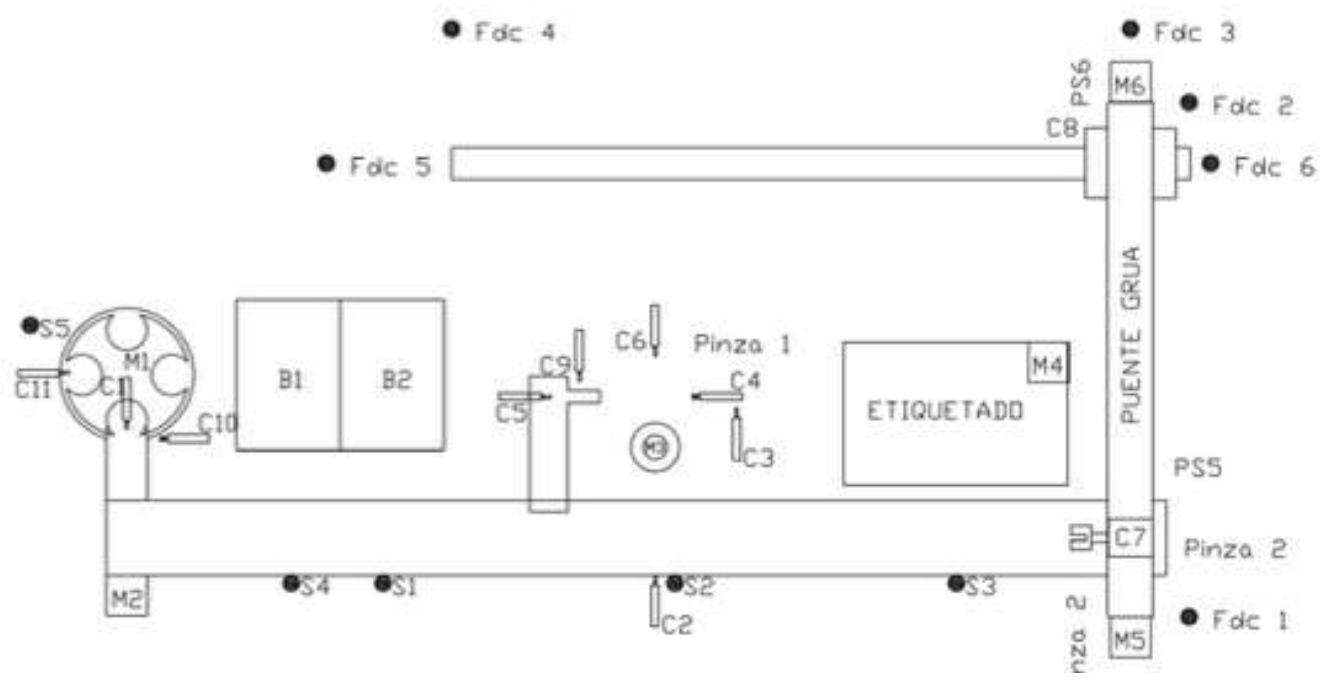

Fig. 3. Ejemplo boceto de un proyecto realizado por los alumnos

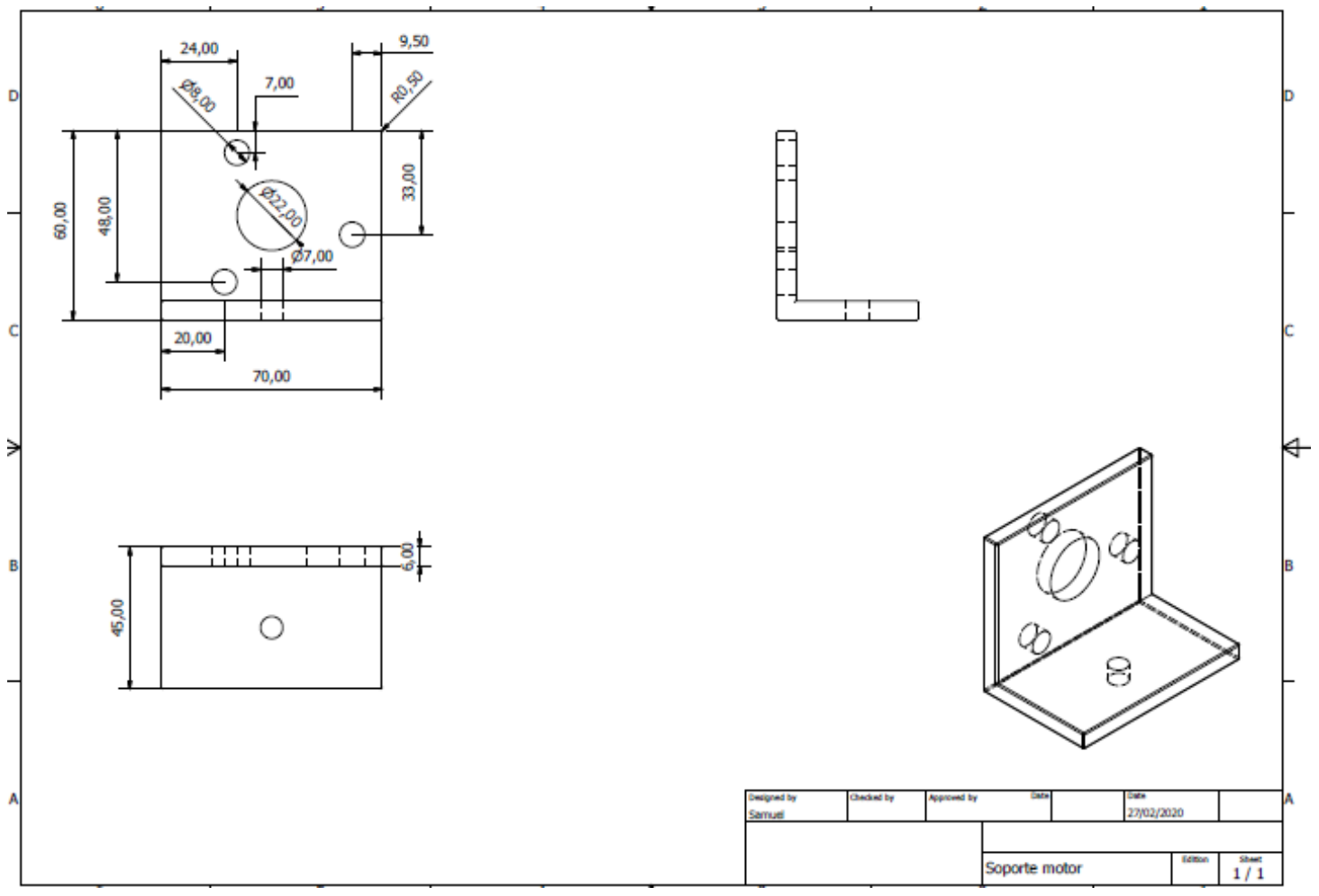

Fig. 4. Ejemplo plano de una pieza realizado por los alumnos 


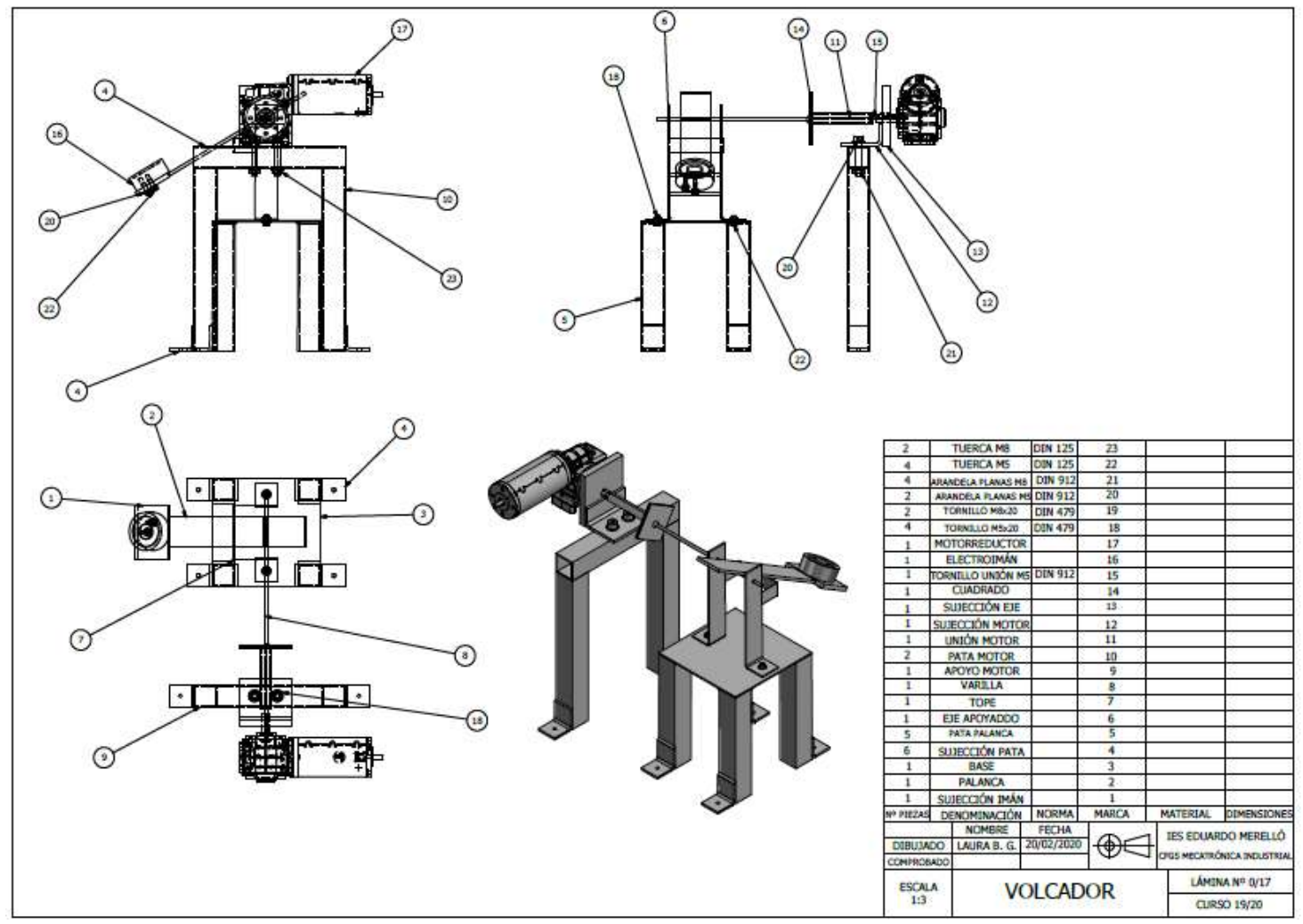

Fig. 5. Ejemplo de plano de conjunto.

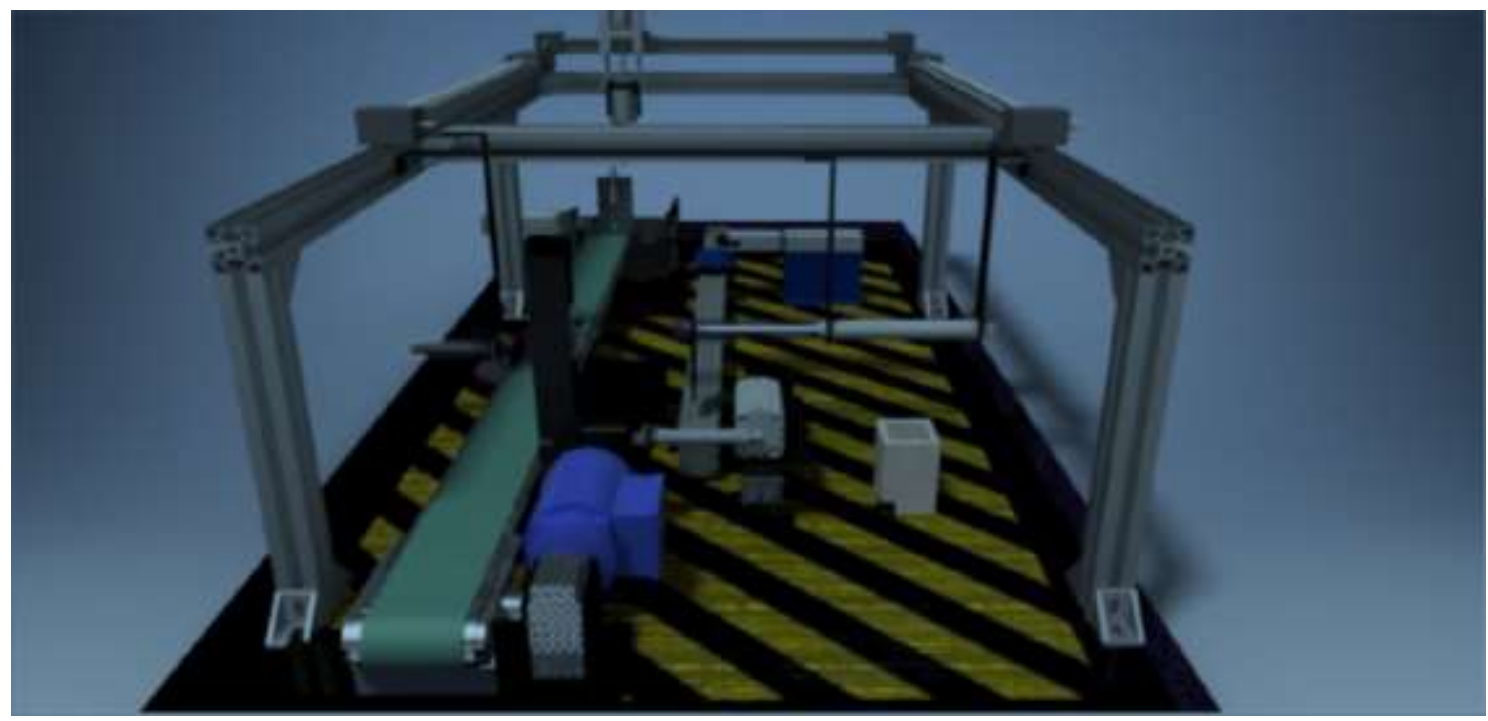

Fig. 6. Ejemplo de renderizado de proyecto final.

\subsection{Realización mecánica}

Dada la falta de experiencia de los alumnos, la realización mecánica no sigue un procedimiento usual en donde habría primero un diseño y después una realización, sino que se realiza prácticamente en paralelo. A pesar de no ser lo usual, se considera que es lo más adecuado según las capacidades de los alumnos.

La realización mecánica comprende diversas operaciones de montaje, mecanizado (torneado, fresado, taladrado), acople de elementos, soldadura, lijado y otros. 
Se adjuntan algunas fotos para ilustrar los procesos anteriores:

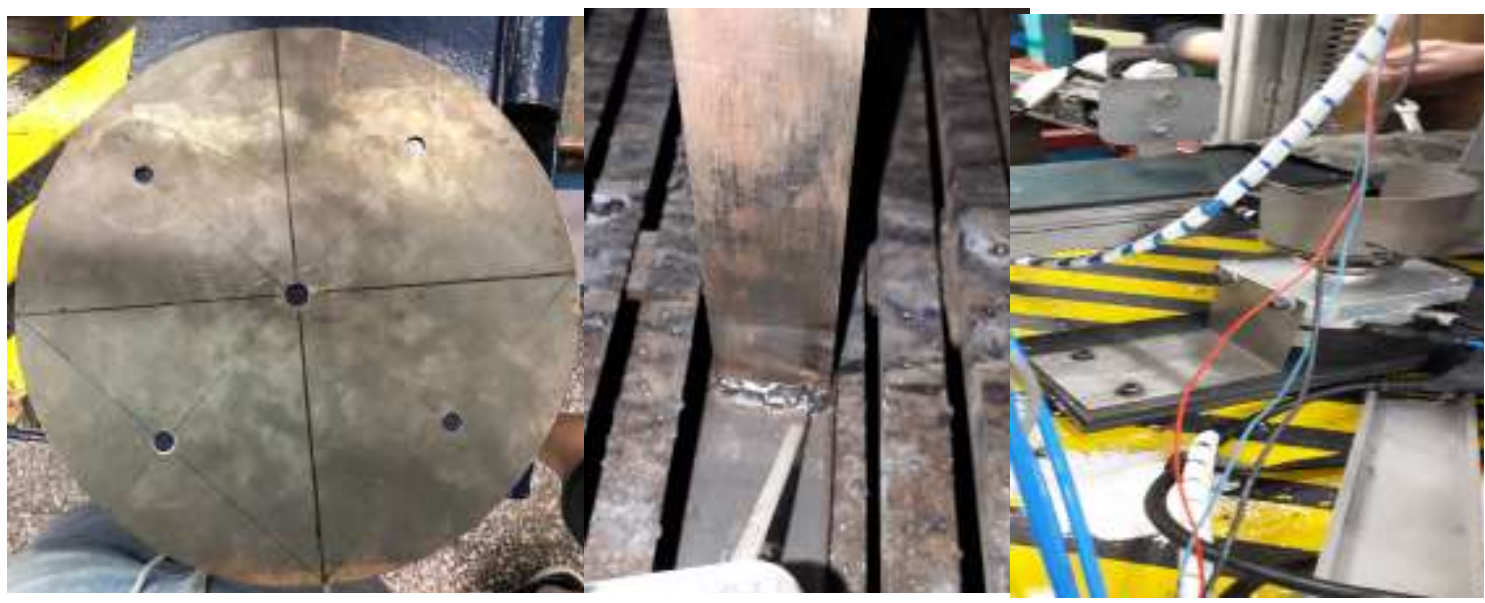

Fig. 7. Fotografias de diferentes fases de la realización mecánica del proyecto realizadas por alumnos.

\subsection{Diseño eléctrico del proyecto.}

El diseño eléctrico del proyecto comprende el diseño y cableado del cuadro eléctrico con los correspondientes elementos de protección: magnetotérmicos, diferencial, relés, fuente de alimentación, variador de frecuencia (si lo hubiera), guardamotor. Los programas de diseño utilizados usualmente son el SEEelectrical y AutoCAD Electrical, pero podrían ser otros.

Una vez realizado el cuadro eléctrico del proyecto, se precede a cablear el proyecto en sí (teniendo en cuenta el avance en su construcción).

Entre los accionadores eléctricos tenemos motores de corriente continua, normalmente a $24 \mathrm{~V}$, y motores de corriente alterna.

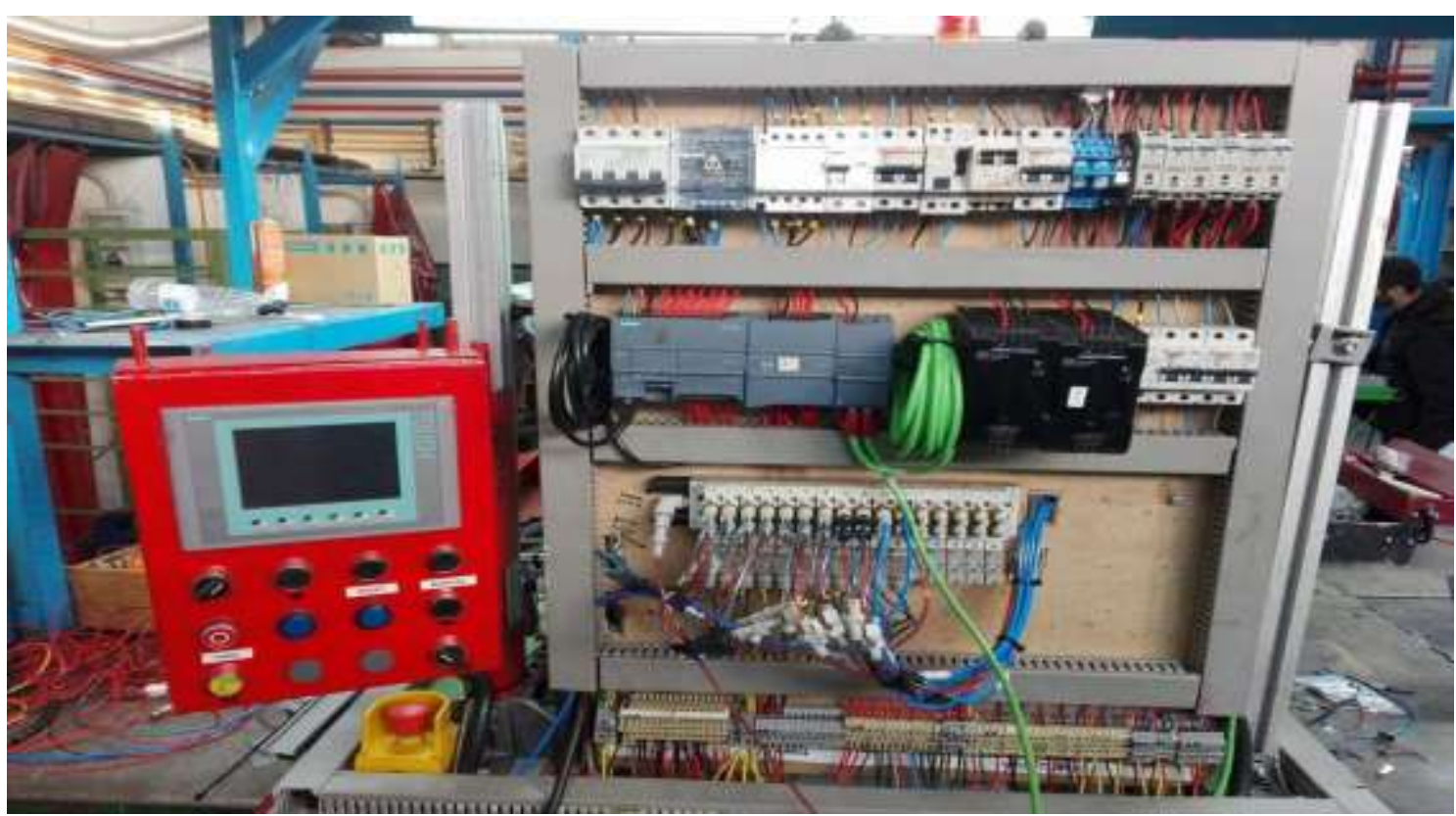

Fig. 8. Ejemplo de cuadro eléctrico realizado por los alumnos. 


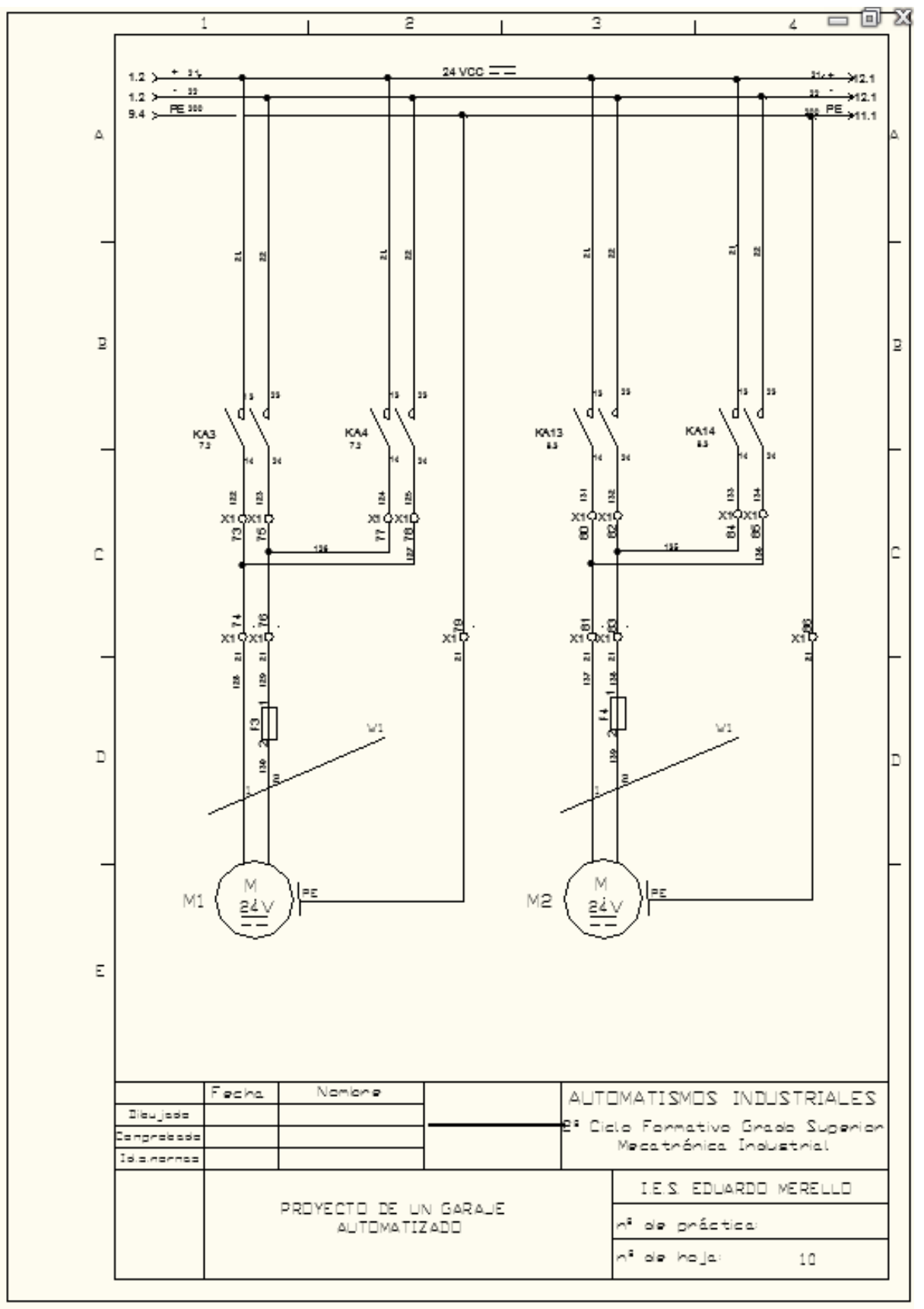

Fig. 9. Ejemplo de plano eléctrico realizado por los alumnos.

\subsection{Diseño neumático}

El diseño neumático es bastante sencillo. Incluye un rack de electroválvulas como el que aparece en la figura 8. Además, habitualmente se utilizan electoválvulas $3 / 2 \mathrm{NO}$, aunque eventualmente se utilizan $5 / 3$ de centros cerrados cuando se quiere mantener alguna posición. Los accionadores habituales son cilindros, pinzas y giradores neumáticos. Normalmente se utiliza el software de diseño Fluidsim pero podría ser otro software. 


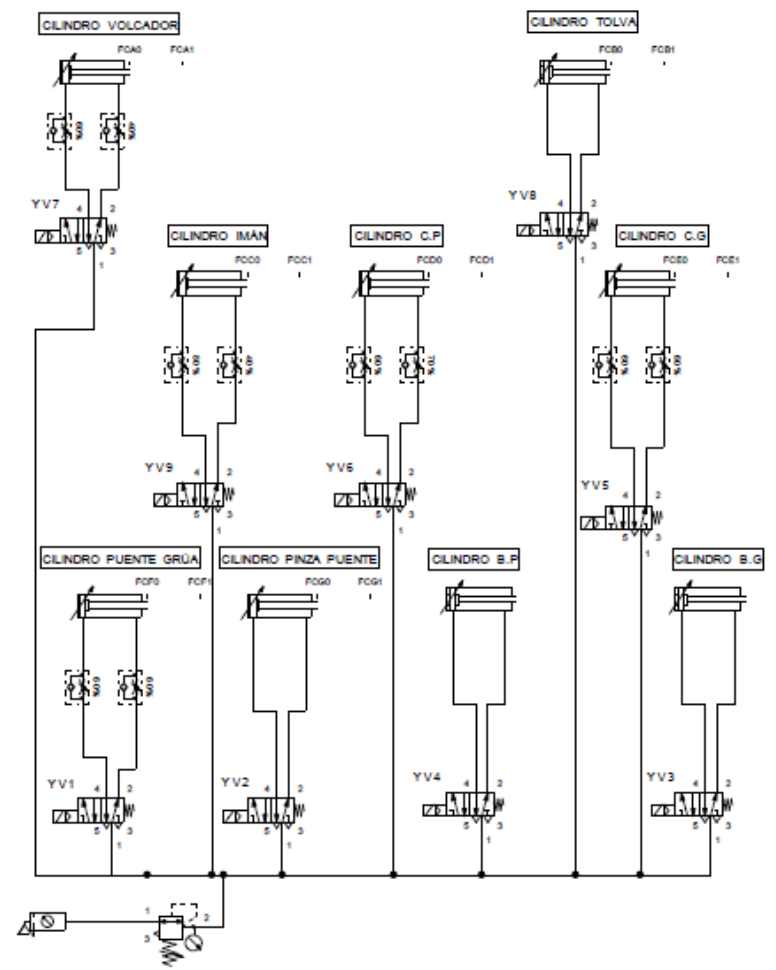

Fig. 9 Ejemplo de plano neumático realizado por los alumnos.

\subsection{Diseño automático}

Para realizar el control se utiliza un autómata S7-1214 AC/DC/Relay, con extensiones de entradas salidas (se puede ver en la figura 8). Que sea la entrada en alterna, nos permite no depender de una fuente de alimentación de corriente continua y que podamos conectar directamente a la red monofásica. La entrada de sensores es a 24VC. Esto, aunque nos limita en flexibilidad, nos garantiza menos problemas de mezclado de líneas y que podamos utilizar un neutro común. Las salidas también son a $24 \mathrm{VC}$ para evitar confusiones y facilitar el cableado.

Los sensores son todos NA y PNP, la mayoría son tipo REED, pero también se utilizan inductivos, capacitivos y reflexivos. Todos funcionan a 24V. Del mismo modo, las señales son todas digitales. Solo excepcionalmente se prevé utilizar sensores analógicos.

También se prevé la utilización de contadores rápidos, empleando encóders para controlar movimientos lineales tipo los que se producen en el símil de puente grúa que se puede ver en la figura 6 . 


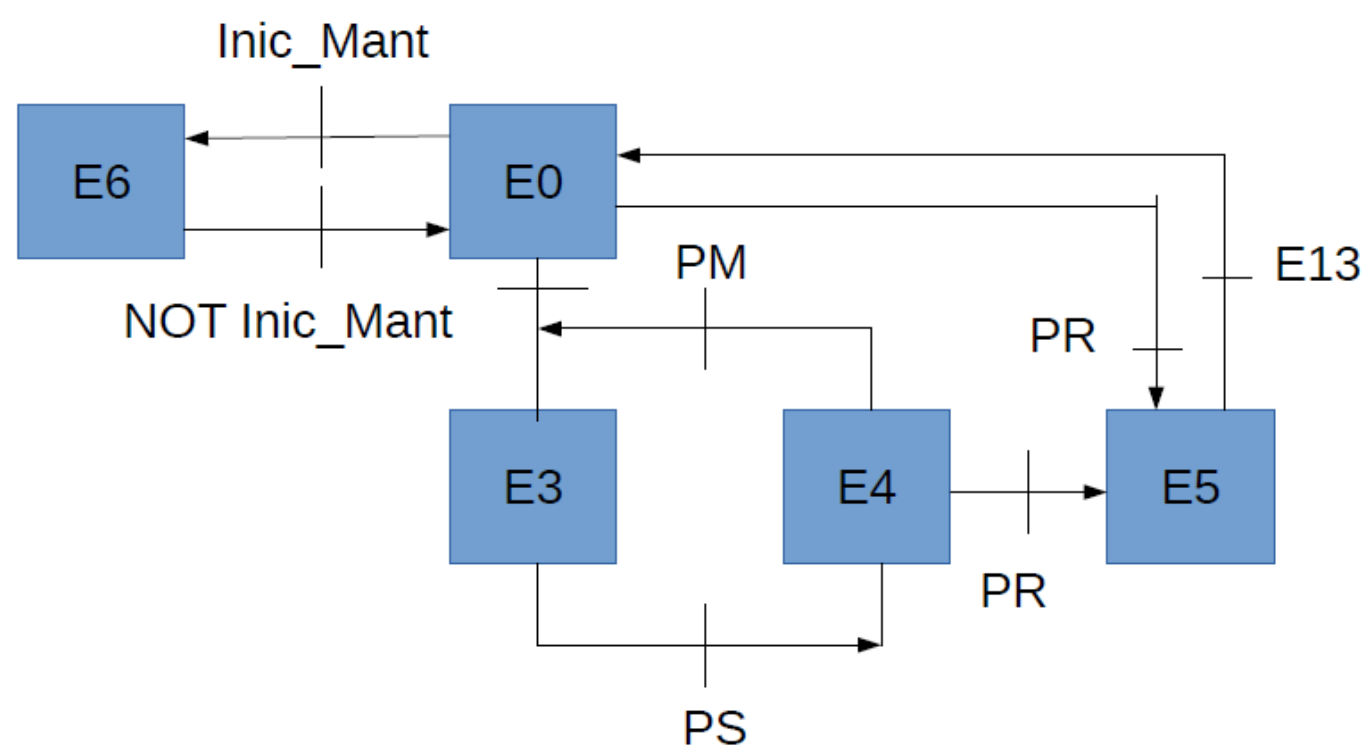

Fig. 10. Grafcet principal o de control del proyecto.

Aunque no es el objetivo de este documento comentar en detalle el diseño neumático, sí que queremos comentar los modos de funcionamiento previstos. La etapa 0 (E0) de la figura 10 secorrespondería con la primera etapa, en donde solo se arranca si se pulsa la marcha (PM) y se dan determinadas condiciones iniciales. Si no se dieran, pulsando el pulsador de reset (PR) podríamos llevar el proyecto a la etapa 5 (E5) en donde se realiza una vuelta a las condiciones iniciales. La etapa 3 (E3) sería la de funcionamiento normal. A partir de ella, cuando queramos podemos pulsar el botón de pause o stop (PS), volviendo a funcionamiento normal al pulsar PM, retomando la ejecución justo donde nos habíamos quedado. Si vemos que no es posible continuar, podemos pulsar PR llevando el proyecto a condiciones iniciales. Además, disponemos de dos modos más de funcionamiento, el que está reflejado en E6 que correpondería al modo de mantenimiento, donde podemos accionar cada elemento por separado para ver que funciona y el de emergencia. Al oprimir el pulsador de emergencia resetearíamos todas las salidas excepto alguna por seguridad (peso en altura u otros).

\subsection{Gestión del Mantenimiento}

Como parte de la gestión del mantenimiento se realiza un AMFE (análisis modal de fallos y efectos), para ayudar en las reparaciones futuras de la máquina, y un plan de mantenimiento preventivo, con las oportunas revisiones que se debieran efectuar. 


\begin{tabular}{|c|c|c|c|}
\hline $0^{\circ}$ & $\begin{array}{l}\text { Gama de mantenimiento } \\
\text { preventivo }\end{array}$ & $\begin{array}{c}\text { Frecuencia } \\
: \text { trimestral }\end{array}$ & $\begin{array}{l}\text { Codigo } \\
\text { gamma: } \\
\text { CW2019 }\end{array}$ \\
\hline CA & \multirow{2}{*}{ Inspección general diaria } & Edioión: 0 & ESP:PREV \\
\hline & & \multicolumn{2}{|c|}{ Fecha:t6zan: Hoja:3/4 } \\
\hline \multicolumn{4}{|c|}{ Instalación a revisar: ársa cloctrica } \\
\hline \multicolumn{2}{|c|}{$\begin{array}{c}\text { Operario: Adrián marti gimeno } \\
\text { Hore inioio: } 8: 00\end{array}$} & \multirow{3}{*}{\multicolumn{2}{|c|}{$\begin{array}{l}\text { Tiempo normal: } 2 \text { horas } \\
\text { Equipo de protecoión }\end{array}$}} \\
\hline Hora inioio: 8.00 & Hora final: $10: 00$ & & \\
\hline \multicolumn{2}{|c|}{ Herramientas } & & \\
\hline \multicolumn{2}{|c|}{ visual } & \multicolumn{2}{|c|}{ Botas de seguridad } \\
\hline \multicolumn{2}{|c|}{ Riesgoes del trabajo: caidas al mismo nivel } & \multicolumn{2}{|c|}{ Firma del operario } \\
\hline \multicolumn{2}{|c|}{ Materiales } & \multirow{2}{*}{\multicolumn{2}{|c|}{\begin{tabular}{|c|} 
Codigo del material \\
Datos obtenidos
\end{tabular}}} \\
\hline Equipo & Descripción & Datos of & \\
\hline $\begin{array}{l}\text { bobinas de las } \\
\text { electrovastmutas }\end{array}$ & $\begin{array}{l}\text { Comproberemos el estado } \\
\text { del cuerpo protector como } \\
\text { los torrilios se conexión }\end{array}$ & \multicolumn{2}{|c|}{$\begin{array}{l}\text { No encontramos ningìn } \\
\text { defecto ni en los tomilos } \\
\text { de conesión ni en al } \\
\text { cuerpo de la bobina }\end{array}$} \\
\hline cableado & $\begin{array}{c}\text { Comproberemos ef estado } \\
\text { del conductor( roturas del } \\
\text { sislamierto) }\end{array}$ & \multicolumn{2}{|c|}{$\begin{array}{l}\text { No encontramos ningin } \\
\text { delecto en los } \\
\text { conductores }\end{array}$} \\
\hline
\end{tabular}

Fig. 11. Ejemplo del plan de mantenimiento preventivo realizado por los alumnos

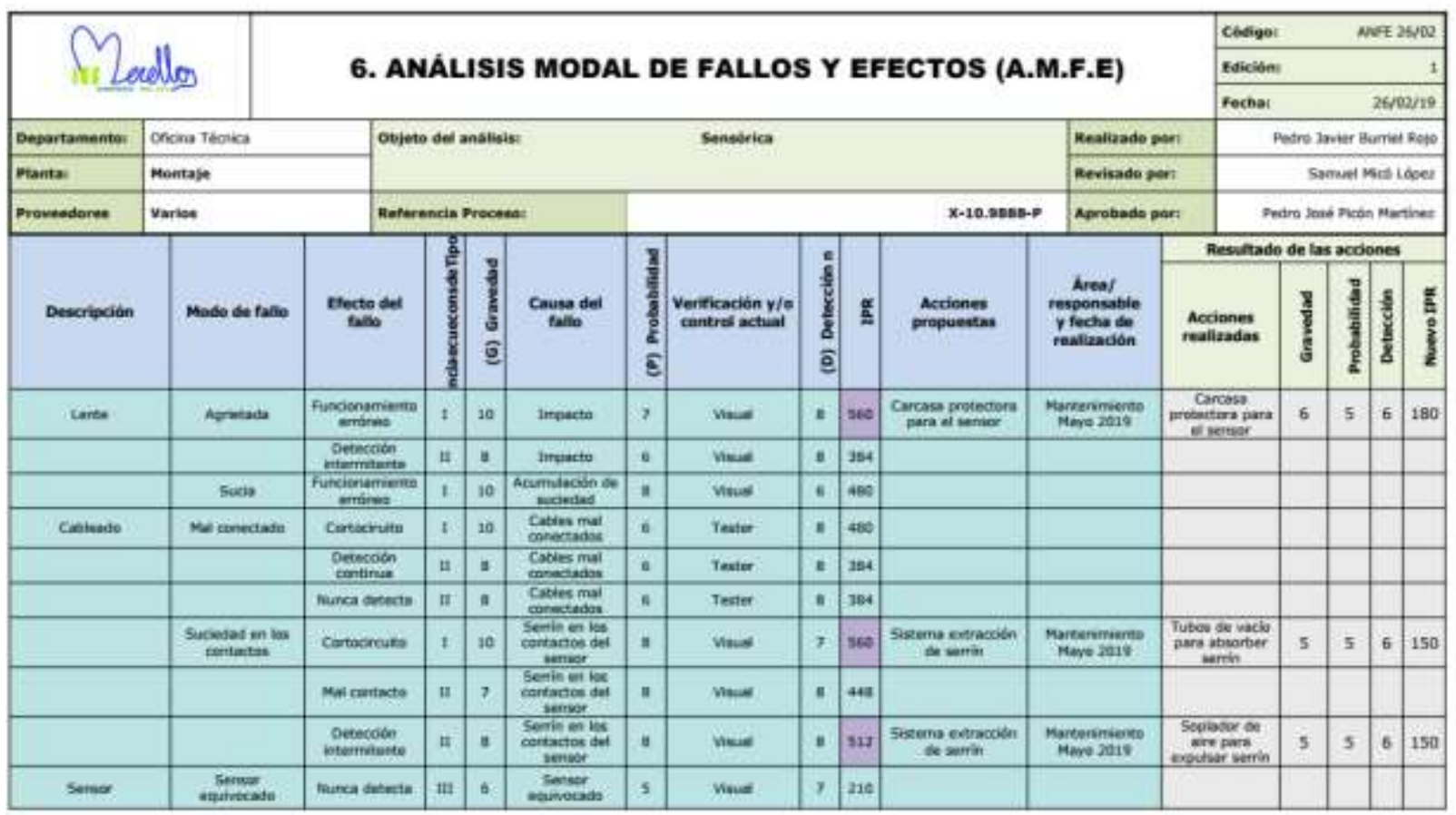

Fig. 12. Ejemplo de AMFE realizado por los alumnos

\section{Resultados}

Los resultados son un proyecto totalmente funcional que integra las diferentes capacidades del ciclo formativo comentadas en la introducción. Además, se ha trabajado la capacidad de planificación de los alumnos, así como la necesidad de coordinación entre ellos, haciendo frente a un desafío complejo con una fecha limitada de entrega. Como ejemplo,puede verse el video Perfomatic en el siguiente enlace: https://www.youtube.com/watch?v=-JXh7co-qn0 
Los proyectos se exponen en una jornada de puertas abiertas a las familias, el resto de los alumnos, autoridades y a la sociedad en general, aumentando todavía más la recompensa emocional al gran trabajo realizado. Además, se realiza un video que se cuelga en internet en la web del instituto y en el canal del profesor responsable.

\section{Conclusiones}

El proyecto constituye un reto que al principio parece inalcanzable para los alumnos que ven simplemente un tablón de madera vacío. Pero poco a poco, con trabajo y la ayuda del profesor, consiguen darle forma.

A pesar de las limitaciones de tiempo y material, se considera un ejemplo de trabajo adaptable a otros ciclos formativos de la misma familia y también incluso como idea general a ciclos de distintas familias.

El aprendizaje activo genera conocimiento significativo, y con posterioridad al proyecto los alumnos se encuentran mejor preparados para integrarse el mundo laboral pudiendo demostrar que ya participaron de la concepción y construcción de un proyecto complejo, debiendo tomar decisiones en entornos con una determinada incertidumbre e innovar para resolver problemas.

Desde el punto de vista del profesor, es un reto el coordinar distintos proyectos con alumnos nuevos cada año que integran conocimientos que por su diversidad no es fácil dominar. En esta situación siempre se puede recurrir al consejo de otros compañeros o incluso de comerciales o expertos de empresas suministradores, que es en definitiva lo que ocurre en el entorno real.

\section{Agradecimientos}

Agradecer a los alumnos su implicación en el desarrollo de los proyectos. Muchos de los retos fueron idea suya, como también lo son la totalidad de figuras del presente artículo. Buena parte de ellos han acudido al laboratorio fuera de horas lectivas, a lo que habría que añadir las innumerables horas que han dedicado en sus propias casas. Sin su esfuerzo la realización de los proyectos no sería posible.

También agradecer a la dirección del IES Eduardo Merello su paciencia cuando se han ocupado espacios, pedido material prácticamente fuera de tiempo o presupuesto y todavía más a los compañeros que año tras año colaboran con consejos participando directamente en el proyecto, también por la paciencia que tienen cuando les ocupamos espacios de taller.

\section{Referencias.}

BELL, S. (2010). "Project-Based Learning for the 21st Century: Skills for the Future" en The Clearing House, vol. 83, issue 2, p. 39-43, DOI: 10.1080/00098650903505415

BLUMENFELD, Phyllis C., et al. Motivating project-based learning: Sustaining the doing, supporting the learning. Educational psychologist, 1991, vol. 26, no 3-4, p. 369-398.

CARNEIRO, R. (2007). "The big picture: understanding learning and meta-learning challenges" en European Journal of Education, vol. 42, issue 2, p. 151-172.

España, Real Decreto 182/2008, de 8 de febreo, de 2008, por el que se complementa el Catálogo Nacional de Cualificaciones Profesionales. BOE, 22 de febrero de 2008, núm. 46, p. 10422 a 10536.

España, Real Decreto 1576/2011, de 4 de noviembre, de 2011 por el que se establece el Título de Técnico Superior en Mecatrónica Industrial y se fijan sus enseñanzas mínimas.. BOE, 10 de diciembre de 2011, núm. 297, p. 131008131079.

España. Comunidad Valenciana. Orden 31/2015, de 13 de marzo de 2015, de la Consellería de Educación, Cultura y Deporte, por la que se establece para la Comunitat Valenciana el currículo del ciclo formativo de grado superior 
correspondiente al título de Técnico Superior en Mecatrónica Industrial. DOGV, 25 de marzo de 2015, núm. 7482, p. 8742-8773.

"Perfomatic". Youtube $<$ https://www.youtube.com/watch?v=-JXh7co-qn0 $>$ [Consulta: 4 de abril de 2020]

SCOTT, C.L. (2015). The Futures of Learning 2: What Kind of Learning for the 21st Century? EDUCATION RESEARCH AND FORESIGH 14, November 2015. United Nations. Educational, Scientific and Cultural Organization working papers. 